\title{
Printed Microwave Frequency Humidity Sensor Operating with Phase Shifting Scheme
}

\author{
Guy Ayissi Eyebe, Benoît Bideau, Éric Loranger, Frederic Domingue
}

\begin{abstract}
This paper investigates a shifting sensing scheme combining slots, transmission lines, and printing technologies. This sensing scheme translates the electrical sensitivity of a transmission line conductor to the insertion phase as a measurement variable. A coplanar waveguide (CPW) based structure was designed, screen-printed, and tested on relative humidity (RH) conditions ranging from $22.8-75.3 \%$ RH. For the first time, a composite material made of poly-pyrrole and TEMPO Oxidized Cellulose Nanofibers (TOCN/PPy) was integrated to the structure and studied as a humidity sensitive conductor in microwave frequencies. The measured sensitivity was $0.154 \%$ RH at $5.870 \mathrm{GHz}$, while insertion losses decreased by $1.26 \mathrm{~dB}$. The effects of sensing layers thickness as well as trade-off considerations between phase sensitivity and signal attenuation were analyzed by simulation.
\end{abstract}

Index Terms-RFID, Screen-Printing, RH Sensing, TOCN/PPy.

\section{INTRODUCTION}

$\mathrm{P}$ rinting technologies have been involved intensively in a bid of improving the competitivness of wireless sensors considering cost, performance, and ease of implementation. These improvements impacted fields like the Internet of Things, industrial safety, and automotive systems [1-4]. In that respect, various sensing schemes imparted from printing materials properties have been proposed. Each material type was investigated for its specific properties in the perspective to develop highly sensitive, low hysteresis and fast response printed sensors. Such materials include dielectric, semi-conductive and conductive inks, as well as dielectric sheets [5-8].

First, some dielectric sensitive substrates have been studied. A study investigating Kapton polyimide foil as a sensing substrate for relative humidity $(\mathrm{RH})$ variations has been proposed [9]. Achievable sensing schemes in $\mathrm{RF} /$ microwave frequencies while using the coplanar waveguide (CPW) transmission line technology were identified. The underlying mechanism allowing sensing principle fulfilment translated the substrate dielectric sensitivity to the effective permittivity variation as a function of RH. Measurement variables in frequency and phase shifting paradigms included the resonant frequency and the insertion phase [10].

Dielectric inks have been involved in implementing sensing applications with printed microwave devices as well. Dielectric inks can enclose sensitive compounds or act as sensitive materials themselves. Locally dispensed, they correlate sensors electrical properties to the environmental parameter of interest, e.g. humidity, pressure, gas concentration or temperature. In that respect, inkjet printing of Barium Strontium Titanate (BST) in phase array applications has been considered for temperature sensing $[11,12]$. Polyvinyl alcohol (PVOH) has been deposited on microwave sensing devices to improve RH sensitivity [13]. Measurement variables belong to phase and frequency shifting patterns, as in the case of sensitive sheets.

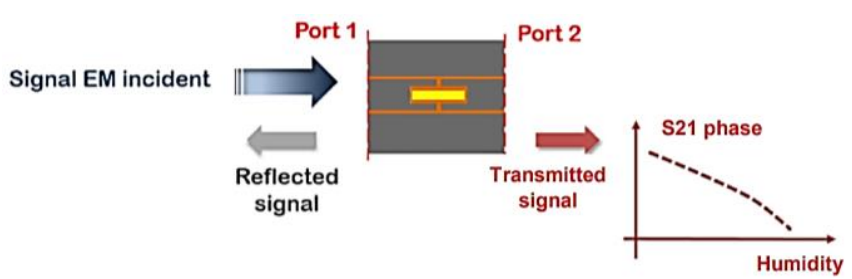

Fig. 1: The phase shifting scheme with transmission line circuits

Finally, conductive and semi-conductive inks have been involved to allow microwave printed devices assess environmental parameters in power variation pattern. An inkjet-printed and chip-less sensor has been reported to detect $\mathrm{CO}_{2}$ and temperature variations with a single-walled carbon nanotube (SWCNT) based ink [14]. A stub resonator loaded by a multi-walled carbon nanotube (MWCNT) strip has been added to integrate harmful gases detection as well [15]. Other conductive sensitive inks such as graphene and PEDOT: PSS have been studied to address sensing applications [16, 17]. A sensing approach to detect a predefined power limit based on a RFID tag power emission has also been reported [18].

While dealing with printed sensors in power variation and frequency-shifting schemes, reading and processing circuitry are located remotely. Using sensitive substrates, the characteristic impedance will change with permittivity, thereby inducing undesirable frequency shifts and impedance mismatches over the whole RF chain. On the other hand, RF power assessment requires remote antennas or inductive coupling. To overcome such limitations, transmission lines technologies are appealing in that they avoid the requirement of remote interrogation. Moreover, they allow simple integration of sensitive conductor and offer excellent design flexibility to implement sensing functions. From authors' best knowledge, there is no study on printed RH sensors operating with a phase shifting scheme, using transmission lines so far.

This paper investigates a phase-oriented sensing scheme allowed by an original combination of transmission line technologies, slots, and printable sensitive conductors. Such an approach might help to integrate sensing abilities to microwave systems involving phase-based systems (See Fig. 1). At least two clues can be considered. First, the electrical length variation of the transmission line, as the sensitive element conductivity changes with the parameter of interest. Switched line or loaded line phase shifters are concerned $[19,20]$. Secondly, the input impedance and phase constant variations might be thought. Such a strategy applies to reconfigurable systems for example [21]. The proposed sensing scheme then allows applying printing technologies to such systems, taking advantage of their lowcost potential, and the full range of sensing materials convenient to address sensing applications. 


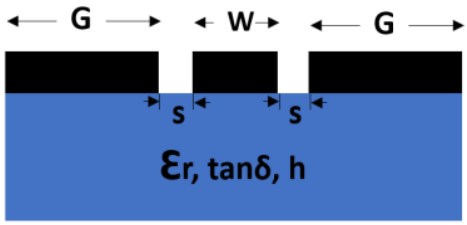

(a)

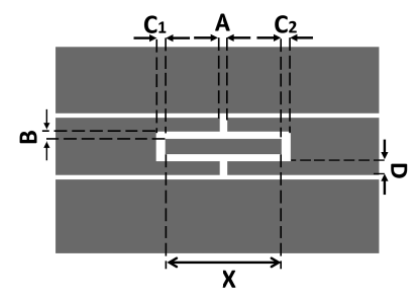

(b)

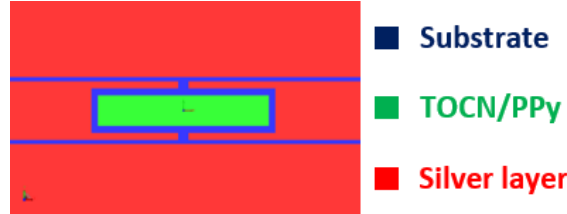

(c)

Fig. 2: Structural parameters of the studied CPW-based structure (a) Geometry of the CPW line; (b) Dimensions of the slots delineating the inner rectangle and (c) Materials type and location on the CPW-based structure.

CPW transmission line technology was chosen for having low dispersion, exhibiting a planar configuration, and making it possible to prototype microwave devices with only one single layer deposition. This could further be achieved when involving the screen printing technique in the fabrication process [9]. CPW supports electromagnetic (EM) signals propagation in nearly transverse electric and magnetic (quasi-TEM) mode. Slots were inserted to decouple electrical properties of the CPW line and that of the sensitive conductor. A composite made of TEMPO Oxidized Cellulose Nanofibers (TOCN) and poly-pyrrole (PPy) is for the first time studied as sensitive material for $\mathrm{RH}$ sensing applications in microwave frequencies.

The geometry, the operation, and the lumped elements modelling of the CPW-based structure to be investigated are first presented in section II. The sensing principle and trade-offs are explained and simulated in section III. Section IV presents design considerations applicable to the sensing material layer. The screen-printing of the device is detailed in section $\mathrm{V}$, meanwhile sections V, VI and VII present experimental section, discussion and comparison respectively.

\section{THE CPW-BASED MicrowaVE StRUCTURE GEOMETRY, OPERATION AND MODELLING}

\section{A. $\quad C P W$-Based Structure Geometry}

The CPW technology exhibits appealing flexibility in the design of microwave devices when combined with adequate slots and discontinuities shapes [22]. The structure studied in this research falls within this category. While the CPW line dimensions were $W=4.16 \mathrm{~mm}$ and $s=0.3 \mathrm{~mm}$, the ground plane width was calculated and set to $G=15 \mathrm{~mm}$ (See Fig. 2 -a). The whole structure was $3.3 \mathrm{~cm}$ in length and $3 \mathrm{~cm}$ in width. The conductive material was silver, one of the most popular materials used for this purpose in printed electronics. The silver layer thickness was set to $10 \mu \mathrm{m}$, based on previous experiments data in our laboratory. Novele IJ-220 sheet was chosen as a substrate. Novele IJ-220 is a PET sheet that went through a chemical surface treatment to ensure proper adherence of printed electronics inks on one side [23]. Given the thinness of chemical coating, only dielectrics properties of PET were considered in the design. The permittivity was set to $\varepsilon_{r}=3.3$, and the dielectric losses to $\tan \delta=0.015$ [24]. The thickness was set to $h=140 \mu \mathrm{m}$ according to Novele IJ-220 datasheet.

Table I: Geometrical parameters of the studied CPW-based structure

\begin{tabular}{cccccc}
\hline Dimensions (mm) & $A$ & $B$ & $C$ & $D$ & $X$ \\
\hline Sensing structure & 0.20 & 0.26 & 0.26 & 0.51 & 7.92 \\
\hline
\end{tabular}

Derived from [25], the geometry of the studied CPW-based structure consists of a rectangle delineated within the central track of the CPW line portion using slots (See Fig. 2-b). The soformed rectangle is named "inner rectangle" below. This geometry confers attractive properties to implement sensing applications. Slots isolate the inner rectangle from the CPW line so that their conductive properties are decoupled. The inner rectangle metallization behaves as a standalone material. Then, some of its characteristics can be involved to integrate sensing capability to printed microwave devices, e.g. electrical conductivity, thermal conductivity, and thickness swelling. Moreover, the substrate was thin enough to allow the structure be sensitive to the variation of the silver conductive layer [26]. This feature favours excellent sensing performance with the CPW-based structure in Fig. 2-b (See Table I gives for dimensions). Impedance and phase variations will be very large.

\section{B. Operation of the $C P W$-Based Structure}

The CPW-based structure in Fig 2-b was proven to operate as a resonator in the literature [25]. One approach to calculate its resonant frequency was proposed in [9], and applied to this research. The theoretical resonant frequency was found to be $f_{r_{t} t h}=5.71 \mathrm{GHz}$. The primary operation of the structure was studied by analyzing EM field distribution as a function of frequency, with ADS simulations. Fig. 3 depicts the results at three frequency points: far from the resonance $(8.5 \mathrm{GHz})$, near the resonance $(6.0 \mathrm{GHz})$ and at the resonance $(5.71 \mathrm{GHz})$.

Referring to Fig. 3-a, it appears from the colour code, the size, and the orientation of EM currents that the EM signals are reflected at the middle of the structure. The slots outlining the inner rectangle left narrow gaps which form serial and fringing effect capacitances, at the edge with the CPW line. Such gap capacitances depend on the operating frequency and the characteristic impedance [26]. Far from the resonance, there is high impedance associated with these capacitances. EM signals are reflected and no signal passes through the structure. However, as the frequency moves toward the resonance, these impedance decreases. The capacitive coupling between the inner rectangle and the CPW line is reinforced. Consequently, EM signals gradually pass through the structure (See Fig. 3-b). At resonance, these impedances are at a minimum. EM signals pass through the structure with maximum intensity and minimum losses (See Fig. 3-c). The symmetry of the structure's geometry induces a symmetry in the frequency response.

Based on the above considerations, a conclusion can be drawn: the frequency-dependent nature of the capacitive coupling makes the structure behaviour dependent on frequency as well. Thus, the choice of the operating frequency is of great 


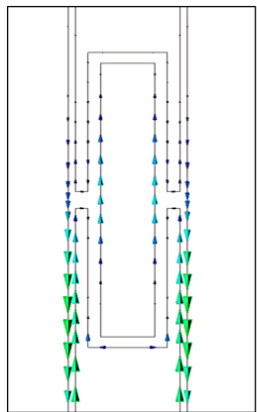

(a)

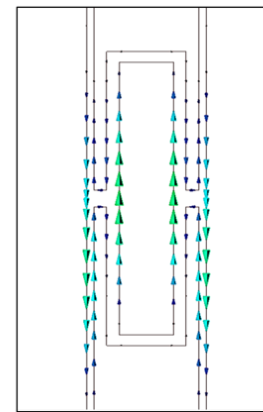

(b)

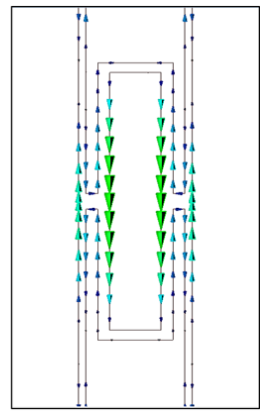

(c)
Fig. 3: EM field currents on the studied CPW-based structure at various frequency points (a) $8.5 \mathrm{GHz}$; (b) $6.0 \mathrm{GHz}$ and (c) $5.71 \mathrm{GHz}$

importance. While phase-shifting schemes requires low insertion loss and high return loss, the operating frequency of the sensing structure should be set at resonance or very close to resonance.

\section{Modelling of the Sensing Structure}

The electrical modelling was an essential step in the research methodology, aiming to find original sensing schemes achievable with the structure in Fig. 2-b. From [27], there are no general closed form equations available for this structure in the literature. However, the influence of the structure geometry and that of each dielectric or conductive material on EM wave propagation can be modelled with the help of adequate lumped elements [28]. This modelling could be achieved thanks to many results reported on previous works in the literature. The half structure to be modelled was taken as an open-end CPW line discontinuity, represented by a capacitance [27]. High-frequency models of the structure involve capacitances and inductances, whose values might fit accurately with polynomial equations taking the inner rectangle length as a variable [29]. Assuming the whole structure as cascade of two serial capacitances, the authors in [9] modelled the structure as a capacitance.

In this paper, the structure was modelled, taking into account its geometry and the inner rectangle metallization conductivity. The result is the network depicted in Fig. 4. The structure geometry is symmetric. Lumped elements modelling the same mechanisms from one side to another are identical [30]. The same inductor represents the additional CPW line portions: $L_{1}$. The capacitive coupling between the CPW line and the inner rectangle is represented by the capacitance $C_{1}$, along with the associated fringing effects. The capacitance $C_{2}$ represents the dielectric properties of the substrate. For their part, $R_{0}$ and $L_{0}$ represent the resistive and the inductive characteristics of the inner rectangle metallization, respectively. The numerical values were extracted from ADS simulation after fitting the lumped elements circuit response to S-parameter simulation. The proposed model was validated at the $5-14 \mathrm{GHz}$ frequency range with minimum discrepancy.

\section{SENSING PRINCIPLE AND TRADE-OFFS}

\section{A. Sensing Principle $C P W$-Based Sensing Structure}

Defining the electrical conductivity of the inner rectangle layer as $\left(\sigma_{C}\right)$, the proposed sensing principle is based on $\left(\sigma_{C}\right)$

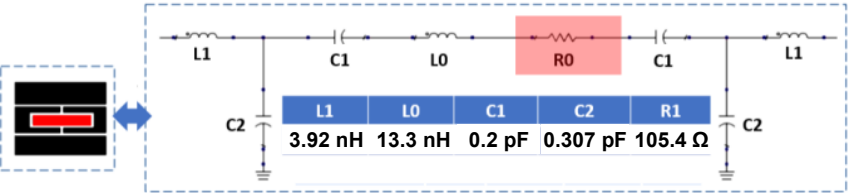

Fig. 4: Lumped elements model of the CPW-based studied circuit

correlation to the environmental parameter of interest. Assumed here to be RH sensitive, $\left(\sigma_{C}\right)$ is named $\sigma_{C}(R H)$ below.

$$
\begin{gathered}
R_{S, C}(R H)=\sqrt{\frac{\omega \cdot \mu}{2 \cdot \sigma_{C}(R H)}} \\
R_{0}(R H)=t \times R_{S, C}(R H) \\
\phi_{21}(R H)=-\arctan \left[\frac{C_{2} \omega}{2-L_{0} C_{2} \omega^{2}} \cdot R_{0}(R H)\right]
\end{gathered}
$$

Any variation of $\mathrm{RH}$ value will be translated onto a variation of $\sigma_{C}(R H)$. From Eq. (1) whereby $\omega$ is the pulsation and $\mu$ the permeability of the propagation medium, the resistivity associated to $\sigma_{C}(R H)$ that is $R_{S, C}(R H)$, will change accordingly. Depending on the layer thickness, $R_{S, C}(R H)$ variation will be reported on the associated resistance that is $R_{0}(R H)$ through Eq. (2) [10]. The expression of the insertion phase $\phi_{21}(R H)$ derived from the frequency analysis of the lumped-elements model in Fig. 4 is given in Eq. (3). It appears that $\phi_{21}(R H)$ depends on $R_{0}(R H)$. Therefore, the $\mathrm{RH}$ value surrounding the printed sensing structure is, as a matter of fact, correlated to the insertion phase. Hence, $\phi_{21}(R H)$ can be used as a measurement variable for RH variation (See Fig. 4). Such a relationship justifies the phase scheme designation of the sensing principle.

The originality of the sensing principle is the ability to translate the electrical properties of sensitive materials to transmission line microwave parameters, namely the insertion phase. The sensing application to be implemented depends on the nature of $\sigma_{C}$. For example, involving temperature-sensitive materials such as graphene will lead to temperature sensing [31]. Likewise, involving hydrogen sensitive material such as palladium will lead to hydrogen sensing [32].

\section{B. FEM simulation of the Sensing Principle}

Given the electrically conductive properties of the printed sensitive element, a FEM simulation was made to investigate the effects of surface current densities on the performance of the phase-oriented RH sensor. The circuit was simulated while operating at its resonant frequency, to create optimal conditions for EM signals propagation. The inner rectangle conductivity was varied up to the conductivity of printed silver layers named $\sigma_{A g}$, by multiple of $10 \%$ steps: $1 \% \cdot \sigma_{A g}, 10 \% \cdot \sigma_{A g}$ and $\sigma_{A g}$. The results are depicted in Fig. 5.

The first observation is derived from the colour code displayed by the inner rectangle for each electrical conductivity value. It appears the EM field intensity increases with the electrical conductivity of the sensitive printed layer. This observation agrees with transmission line theory in the way that insertion losses decrease as the electrical conductivity of the 


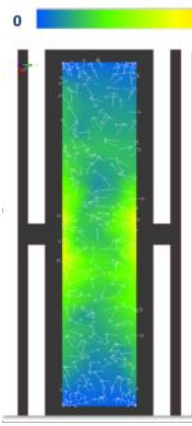

(a)

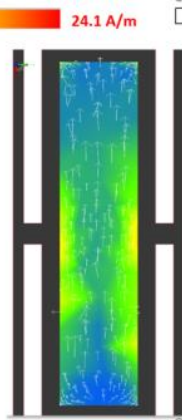

(b)

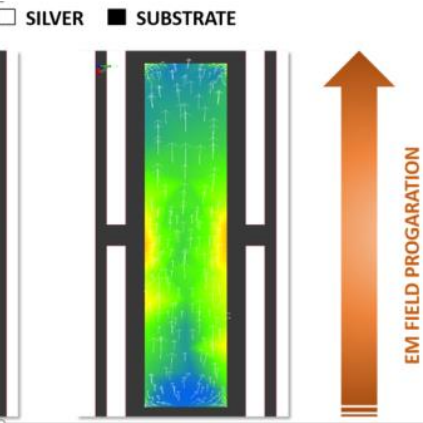

(c)
Fig. 5: Surface current densities on the CPW-based structure at various inner rectangle electrical conductivity values (a) $1 \% \cdot \sigma_{A g}$; (b) $10 \% \cdot \sigma_{A g}$; and (c) $\sigma_{A g}$

conductive layer increases. The corollary is a lowering EM signals reflection and an increase of EM field density passing through the structure. Another remark is the EM field that is mainly located in the centre of the inner rectangle.

The second observation is related to the orientation of surface current density vectors. Far from the resonance, these vectors are randomly oriented (See Fig. 5-a). The hypothesis was made that the weakness of the electric field through the sensing structure can explain this. This weakness is related to the capacitive coupling between the $\mathrm{CPW}$ and the inner rectangle. As the conductivity of the CPW line increases, the capacitive coupling strength increases. EM fields are more easily flared in the inner rectangle, then taking part in polarizing the electrical dipoles on the inner rectangle surface in turn. A positive loop falls into place. As the conductivity get close to the maximum, the surface current density vectors become more strongly oriented in the same direction (See Fig. 5-b and Fig. 5-c). For this reason, one might prioritize sensitive printable materials with high electrical conductivity.

\section{Sensitivity-Attenuation trade-off with the sensing structure}

While sensing RH variation with the structure in Fig. 2-b, one major aspect should be taken into account: the attenuation due to the conductor. Assuming the centre strip was made of an RH sensitive conductive layer, an expression of the attenuation constant of CPW lines $\alpha_{c}(R H)$, is given in Eq. (4) as derived from [26]. $R_{C}(R H)$ is the series resistance in ohms per unit length of the central strip conductor, and $R_{G}$ is the distributed series resistance in ohms per unit length for the ground planes. For this expression to be valid, the assumption is made that the thickness $t$ is far greater than the skin depth $\delta(t>5 \delta)$ [26].

$$
\alpha_{c}(R H)=\frac{R_{C}(R H)+R_{G}}{2 Z_{0}}
$$

$R_{C}(R H)$ and $R_{G}$ are inversely proportional to $\sigma_{C}(R H)$ and $\sigma_{G}$ respectively. The RH sensitive nature of central strip conductivity makes $\alpha_{c}(R H)$ change with RH. Noting that the inner rectangle area is a fraction of the central strip area, $R_{0}(R H)$ is assumed to be a fraction of $R_{C}(R H)$. Thus, in attenuation constant analysis, what applies to $R_{C}(R H)$ also applies to $R_{0}(R H)$. From Eq. (4), the attenuation constant increases with $R_{0}(R H)$.
From a phenomenological viewpoint, the increase of attenuation counteracts the sensitivity. Eq. (3) shows that insertion phase variation increases with $R_{0}(R H)$. It then appears from Eq. (3) and Eq. (4) that in a contradictory way, sensitivity and signal attenuation all result from $R_{0}(R H)$ increasing. Thus, a trade-off consideration appears inherently to the sensing principle. When acting as a phase shifter, the sensing structure should induce a wide phase-shift while keeping insertion losses to a minimum. A figure of merit named $F_{\Delta \phi}$ is used further in this paper to evaluate to what extent the sensitivity is improved without reaching a critical attenuation of EM signals.

\section{Design Considerations With the Phase-OrIENTED CPW RH-SENSOR}

While using conductive and semi-conductive materials as sensitive elements, two main mechanisms might occur after interaction with the environmental parameter of interest: the variation of metallization electrical conductivity or its layer swelling. A study analysing their effects on the sensing principle was done by FEM simulation with EMPro software.

\section{A. Effect of $\sigma_{C}$ and Validation of the Sensing Principle}

The effect of inner rectangle layer conductivity on the sensing principle was investigated by varying $\sigma_{C}$ to $100 \% \cdot \sigma_{A g}$, $10 \% \cdot \sigma_{A g}, 1 \% \cdot \sigma_{A g}$ and $0.1 \% \cdot \sigma_{A g} \cdot \sigma_{A g}$ was set at $2.10^{7} \mathrm{~S} / \mathrm{m}$ based on previous experiments in our laboratory. The silver thickness was fixed at $10 \mu \mathrm{m}$. This value was also found realistic for the screen-printing technique. Simulation results for the $\sigma_{C}$ variation are given in Fig. 6-a to Fig. 6-c. From Fig. 6-a, the insertion phase $\phi_{21}$ was analyzed for $f_{r}=5.70 \mathrm{GHz}$. $\phi_{21}$ decreased from $7.30^{\circ}\left(\sigma_{C}=\sigma_{A g}\right)$ to $4.15^{\circ}\left(\sigma_{C}=1 \% \cdot \sigma_{A g}\right)$. The correlation between the insertion phase and the inner rectangle conductivity validates the sensing principle by simulation.

From Fig. 6-c, three observations are made. The first is the worsening of return loss as $\left|S_{11}\right|$ decreases from $29.66 \mathrm{~dB}$ when $\sigma_{C}=\sigma_{A g}$ to $19.86 \mathrm{~dB}$ when $\sigma_{C}=1 \% \cdot \sigma_{A g}$. This may come from the input impedance variation of the sensing structure. As $\sigma_{C}(R H)$ increases, $R_{0}(R H)$ decreases as detailed in section IIIA. The change in $R_{0}(R H)$ modifies the input impedance of the sensing structure, which is improved when $R_{0}(R H)$ decreases and worsened when $R_{0}(R H)$ increases. The reflection coefficient then changes as a function of the mismatch between the inner rectangle and the CPW line. As a result, $S_{11}$ changes.

The second observation is the resonance shifting from 5.648 $\mathrm{GHz}\left(\sigma_{C}=\sigma_{A g}\right)$ to $5.433 \mathrm{GHz}\left(\sigma_{C}=1 \% \cdot \sigma_{A g}\right)$. The skin depth variation can explain such a situation. The skin depth $\delta$ changes with the inner rectangle conductivity: $0.6 \mu \mathrm{m}$ for $\sigma_{C}=$ $\sigma_{A g}, 1.87 \mu \mathrm{m}$ for $\sigma_{C}=0.1 \cdot \sigma_{A g}$ and $18.7 \mu \mathrm{m}$ for $\sigma_{C}=0.1 \%$. $\sigma_{A g}$. The ratio between conductor and substrate thickness is $h / \delta=233,74.9$ and 7.49 respectively. Given $h / \delta$ value, the characteristic impedance of the sensing structure depends on the conductor thickness [26]. As a result, resonant frequency and transmission phase shifting occur accordingly.

Finally, from Fig 6-a to Fig. 6-c, curves with $0.1 \% \cdot \sigma_{A g}$ disagree with the above-mentioned explanations. The high divergence observed is attributed to the collapsing of the capacitive coupling between the CPW line and the inner rectangle. The 


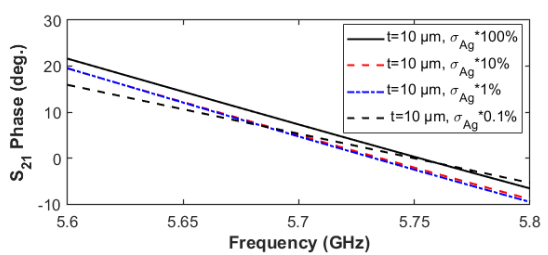

(a)

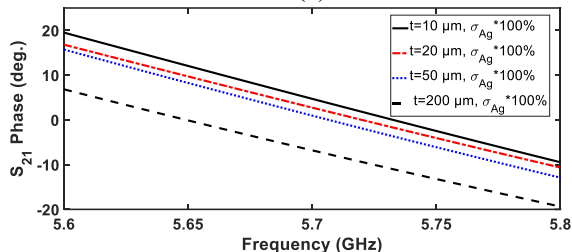

(d)

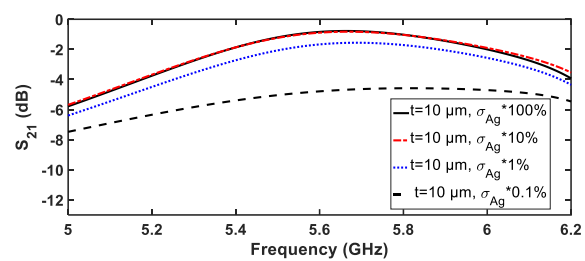

(b)

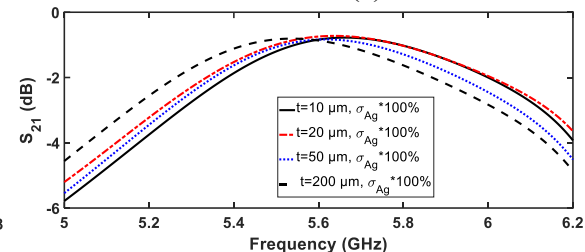

(e)

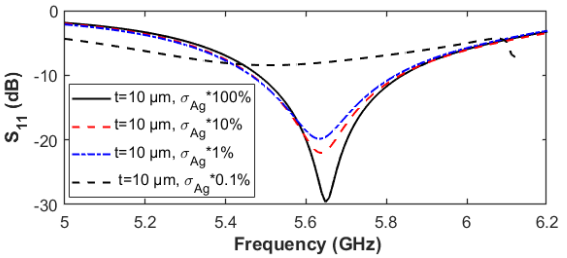

(c)

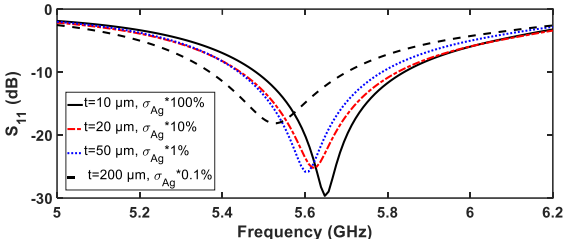

(f)

Fig. 6: Simulated FEM results with the sensing structure (a) $S_{21}$ phase, (b) $S_{21}$ modulus and (c) $S_{11}$ magnitude to evaluate the influence of the sensing layer conductivity variation for $\sigma_{A g}, 10 \% \cdot \sigma_{A g}, 1 \% \cdot \sigma_{A g}$ and $0.01 \% \cdot \sigma_{A g}$, considering a thickness of $10 \mu \mathrm{m}$. And (d) $S_{21}$ phase, (e) $S_{21}$ modulus and (f) $S_{11}$ magnitude to evaluate the influence of the sensing layer thickness variation at $10 \mu \mathrm{m}, 20 \mu \mathrm{m}, 100 \mu \mathrm{m}$ and $200 \mu \mathrm{m}$, considering the conductivity of printed silver $\sigma_{A g}$.

value of the inner rectangle metallization layer is significantly low $\left(\sigma_{C} \ll \sigma_{A g}\right)$ so that no conduction is possible. This hypothesis agrees with the analysis in section III-B. The variation of insertion losses $\left|S_{21}\right|$ are shown in Fig. 6-b from $0.84 \mathrm{~dB}$ $\left(\sigma_{C}=\sigma_{A g}\right)$ to $4.64 \mathrm{~dB}\left(\sigma_{C}=1 \% \cdot \sigma_{A g}\right)$. Decreasing the inner rectangle layer conductivity will lead to a critical value of insertion losses for which EM signals will not be able to pass through the sensing structure.

\section{B. Effect of the Sensitive Layer Swelling}

Some of the sensitive layers increase in thickness after interaction with $\mathrm{RH}$ [9]. The effect of the sensitive layer swelling was studied to help designers and other researchers to take this into account. The conductivity was maintained at $100 \% \cdot \sigma_{A g}$, and the thickness varied to the following values: $10 \mu \mathrm{m}, 20 \mu \mathrm{m}$, $50 \mu \mathrm{m}$ and $100 \mu \mathrm{m}$. The effective dielectric constant of a CPW line as a function of conductor thickness $t$ named $\varepsilon_{e f f}^{t}$, is given in Eq. (5), whereby $K(x)$ is the elliptic integral of the variable $x$. Eq. (6) gives the relation between $\varepsilon_{\text {eff }}^{t}$ and the characteristic impedance $Z_{0}$ for a CPW line.

$$
\begin{aligned}
& \varepsilon_{e f f}^{t}=\varepsilon_{e f f}-\frac{\varepsilon_{\text {eff }}-1}{\frac{W+2 s}{1.4 \cdot t} \frac{K[W /(W+2 s)]}{K\left(\sqrt{1-[W /(W+2 s)]^{2}}\right)}+1} \\
& Z_{0}=\frac{30 \pi}{\sqrt{\varepsilon_{e f f}^{t}}} \cdot \frac{K\left(W+\frac{1.25 \cdot t}{\pi}\left(1+\ln \frac{4 \pi W}{t}\right)\right)}{K\left(\sqrt{1-\left[W+\frac{1.25 \cdot t}{\pi}\left(1+\ln \frac{4 \pi W}{t}\right)\right]^{2}}\right)}
\end{aligned}
$$

The influence of the conductor thickness appears clearly. A change in the layer thickness will induce a change in the characteristic impedance, according to Eq. (5) and Eq. (6). As a result, a resonant frequency shift and a phase transmission variation will be expected. From Fig. 6-f, the resonant frequency shifted from $5.527 \mathrm{GHz}(t=200 \mu \mathrm{m})$ to $5.648 \mathrm{GHz}(t=10$ $\mu \mathrm{m})$. The resonance shift $(121 \mathrm{MHz})$ was higher when compared to the case of electrical conductivity variation $(15 \mathrm{MHz})$ according to theoretical expectations. The peak location of $S_{21}$ shifted with the resonance shifting (See Fig. 6-e). Insertion losses were globally stable around $0.84 \mathrm{~dB}$. There was no significant attenuation, and this was coherent to theoretical expectations given that the conductivity remained unchanged. Moreover, the simulated thickness values were high compared to the skin depth. The phase moved from $-6.81^{\circ}(t=10 \mu \mathrm{m})$ to $5.52^{\circ}(t=200 \mu \mathrm{m})$, which represents a simulated phase shift of $\Delta \phi_{21}=12.33^{\circ}$ at $5.70 \mathrm{GHz}$ (See Fig. 6-d).

\section{FABricAtion of the Phase ORIENTEd PRINTED SENSOR}

The simulated structure was printed circuit through a process with steps including the screen-printing of the microwave structure, the synthesis of the TOCN/PPy sensitive material, and their integration onto one circuit.

\section{A. Screen-Printing of the Microwave Structure}

The Protoprint S apparatus from LPKF was used as screenprinter. A stencil including the negative mask shape of the microwave structure to be printed was purchased from a commercial provider. Other circuits such as Thru-Reflect-Line (TRL) standards intended for microwave calibration and $1.5 \mathrm{~cm}$ square shapes for electrical conductivity measurement were also included. The mask transfer was achieved with the help of a squeegee provided along with the screen-printing kit. HPS021LV silver screen printing ink from Novacentrix (waterbased Ag flake ink, viscosity: $800 \mathrm{cP}$ at $100 \mathrm{sec}^{-1}$, specific gravity: 3.1 and $\mathrm{Ag}$ content: $75 \mathrm{wt} \%$ ) was used. Once transferred, the whole circuit was placed in an oven at $118{ }^{\circ} \mathrm{C}$ during 3 hours, for thermal sintering (see Fig. 7). The sintering temperature was chosen lower than PET glass transition temperature. After that, the circuit was ready for experiments.

As microwave parameters were depending on the circuit flexion, a rigid backing was added to stabilize the circuit, prevent bending and ensure the robustness of the measurement (see Fig. 8-a without backing and Fig. 8-b with backing). 


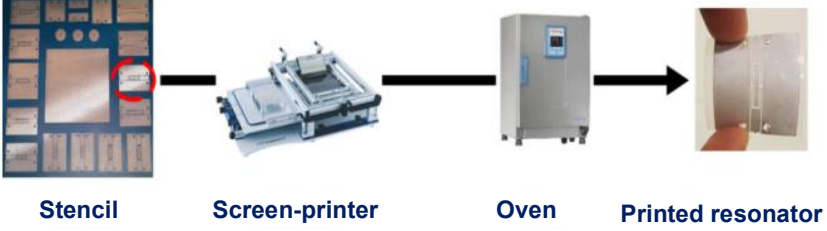

Fig.7: Screen-printing process of the sensing structure

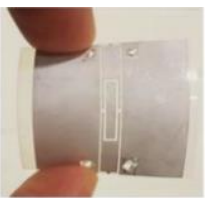

(a)

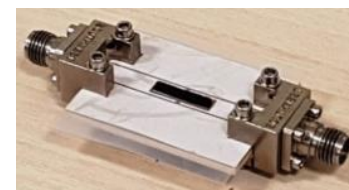

(b)
Fig. 8: Pictures of the printed structure (a) before TOCN/PPy integration, and (b) after TOCN/PPy integration.

Physical and microwave parameters of the testing device were measured after fabrication. The resistivity of the printed silver tracks was extracted based on the 4-probe method results, at $2.07 \mu \Omega . \mathrm{cm}$. This resistivity is 1.33 times the resistivity of bulk silver. The thickness of the silver layer measured with NT1100 optical profilometer from WYKO was $7 \mu \mathrm{m}$. The measured resonant frequency was $f_{r}=5.870 \mathrm{GHz}$. The measured phase transmission was $\phi_{21}=178.7^{\circ}$, and the measured insertion losses were $I . L=4.42 \mathrm{~dB}$.

\section{B. Synthesis of the Poly-Pyrrole Sensitive Material}

PPy has demonstrated many advantages for sensing applications, including good linearity, low hysteresis, fast response and high environmental stability [33]. Its RH sensitive properties have been studied in the literature, with various composites. Mixing strategies included PPy incorporation by cellulose [34], blending with other sensing materials such as graphene [35], and PPy grafting [36]. Chemical oxidative polymerization was also considered to enhance PPy electrical conductivity [37]. From our best knowledge, the highest conductivity of PPy was reported at $150 \mathrm{~S} . \mathrm{cm}^{-1}$, obtained with polystyrene sulfonate (PSS) in a ratio of 0.25 relatively to Pyrrole monomer [38].

Fig. 10 presents the synthesis process of the TOCN/PPy composite that was realized in our chemical laboratory. The preparation started with stirring $80 \mathrm{ml}$ of a TOCN solution at $0.5 \%$, and $2 \mathrm{ml}$ of a $0.028 \mathrm{~mol}$ Pyrrole solution in a beaker for 10 minutes. The iron chloride oxidant solution $\left(\mathrm{FeCl}_{3}\right)$ at $0.5 \mathrm{~mol} / \mathrm{L}$ was added after that. The mixture was kept under stirring for an additional 30 minutes before the polymerisation was stopped. The suspension was then filtered and rinsed thoroughly with distilled water. The film obtained was immersed in hydrochloric acid $(\mathrm{HCl})$ solution at $0.1 \mathrm{~mol} / \mathrm{L}$. $\mathrm{HCl}$ doped the PPy chains with chloride ions and conferred its conductive properties to the film. Finally, the TOCN/PPy film was heated in a sandwich between two hot plates for drying, at $94^{\circ} \mathrm{C}$ for 30 minutes. The final thickness of the film was around $200 \mu \mathrm{m}$.

\section{Integration of TOCN/PPy Film and Microwave Structure}

The TOCN/PPy composite film was stuck on top of the inner rectangle, using a very thin layer of PVOH as glue (See Fig. 11). S-parameters were measured to evaluate the influence of

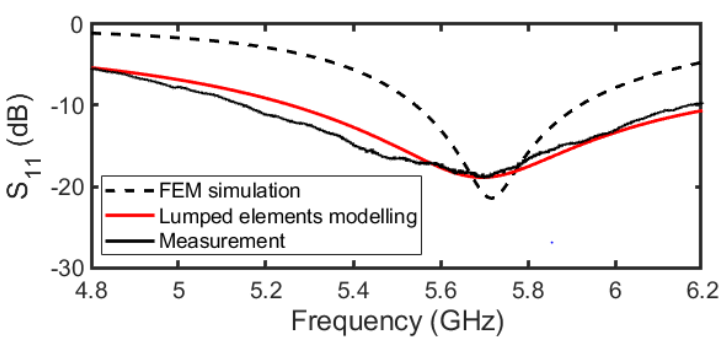

(a)

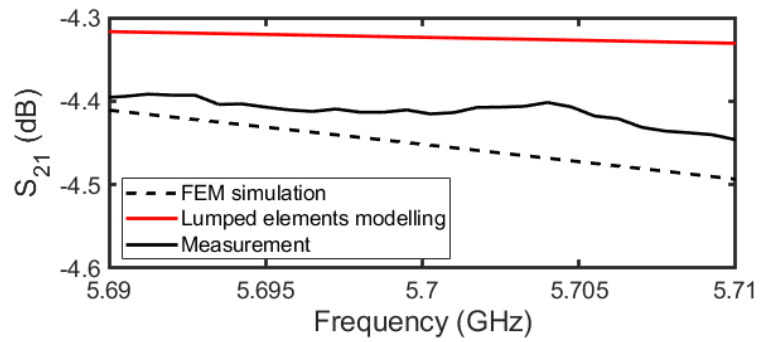

(b)

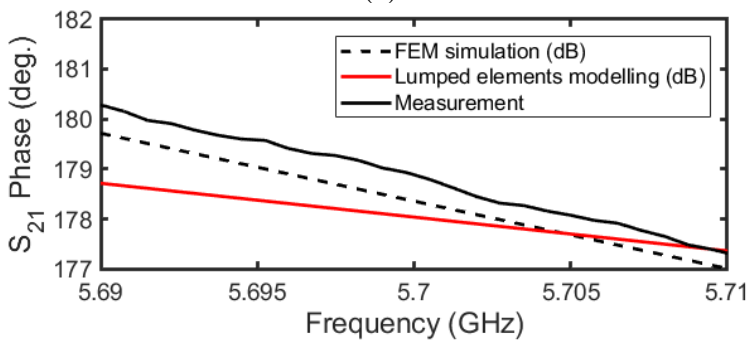

(c)

Fig. 9: Comparison of FEM simulation, lumped elements modelling and measurement of the sensing structure for the following parame-

ters: (a) $S_{11}$ magnitude, (b) $S_{21}$ magnitude and (c) $S_{21}$ phase.

the TOCN/PPy film integration. The resonant frequency moved from $5.870 \mathrm{GHz}$ to $5.806 \mathrm{GHz}$. This variation was attributed to the thickness variation of the inner rectangle layer material, henceforth formed by silver and TOCN/PPy layers stack-up. The insertion phase moved from $178.7^{\circ}$ to $176.5^{\circ}$. The insertion losses worsened from $4.42 \mathrm{~dB}$ to $4.54 \mathrm{~dB}$, owing to the increase of inner rectangle metallization resistivity. All these variations suggest the testing structure will adequately operate in the frequency shift paradigm as expected.

\section{EXPERIMENTAL AND HUMIDITY RESPONSE}

\section{A. Comparison of modelling, simulation and measurement}

Fig. 9 presents the comparison of lumped elements modelling, FEM simulation and measurement before testing the RH sensing capability.

Resonant frequencies are very close for all these results (See Fig. 9-a). The electrical model in Fig. 4 helped to rebuild the frequency behavior adequately. The hypothesis was made that material settings can explain the tip observed with the FEM simulation. The TOCN/PPy layer was simulated as a fully homogenous piece whereas the raw material was a bit porous and rough. The Q-factor was better in FEM simulation conditions.

The discrepancy in insertion losses was relatively low, up to $1.5 \mathrm{~dB}$ (See Fig. 9-b). This can be explained by precision issues while trying to model the printed structure geometry, which combine intertwined shapes of different electrical conductivity. 

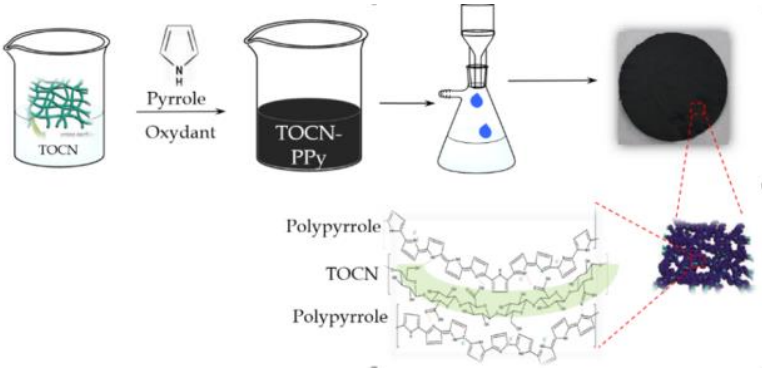

Fig. 10: Process of TOCN/PPy synthesis

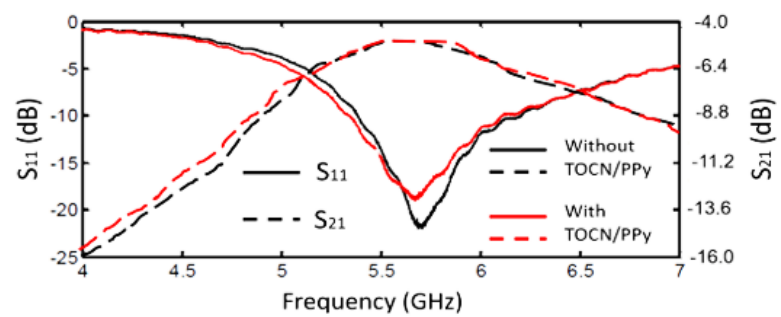

Fig. 11: Measured S-parameters before and after integration of TOCN/PPy

The phase shift difference in FEM simulation and measurements can be explained by the precision on reference planes (Fig. 9-c). Difference with lumped-elements modelling can be explained with the precision of model elements as a function of frequency. However, the maximum gap is around $2^{\circ}$.

\section{B. Humidity Test Setup}

The circuit was tested in fixed $\mathrm{RH}$ levels ranging from $22.8 \% \mathrm{RH}$ (in ambient conditions) to $75.3 \% \mathrm{RH}$. The RH measurement points were generated using aqueous saturated salt solutions [39]. About $100 \mathrm{~mL}$ of each solution was placed inside $4 \mathrm{~L}$ boxes in volume, at $23^{\circ} \mathrm{C}$. The $\mathrm{CPW}$ device was placed inside each box, hanged by cables of the vector network analyzer through small openings (See Fig. 12). The following RH values were generated: $32.8 \% \mathrm{RH}, 43.2 \% \mathrm{RH}, 52.9 \% \mathrm{RH}, 70.8 \% \mathrm{RH}$, and $75.3 \% \mathrm{RH}$. A RH probe was used for real-time RH measurement in the test setup. After the RH value has been settled throughout the box, S-parameters were saved after 3 minutes for each test point. The measurements were taken three times. Only the mean values are represented in the results below.

\section{Humidity Response}

The humidity response of the testing structure was investigated in the perspective of phase-shifting pattern. The insertion phase was studied to evaluate the phase sensitivity. Insertion losses were measured to analyse the attenuation constant, compute and study the phase figure of merit. Return losses were assessed to investigate the impedance matching and the resonant frequency. The curves of $\phi_{21}, S_{21}$ and $S_{11}$ for each RH mentioned above test point are presented in Fig. 13.

Fig. 13-a gives $\phi_{21}$ variation with RH. All the measurements were taken at the frequency of $5.72 \mathrm{GHz}$, where insertion losses were $5.629 \mathrm{~dB}$ at $22.8 \%$ RH. From Fig. 13-b, it appears that $\phi_{21}$ moved from $176.5^{\circ}$ at $22.8 \% \mathrm{RH}$ to $168.1^{\circ}$ at $75.3 \% \mathrm{RH}$. This result experimentally validates the phase shifting sensing

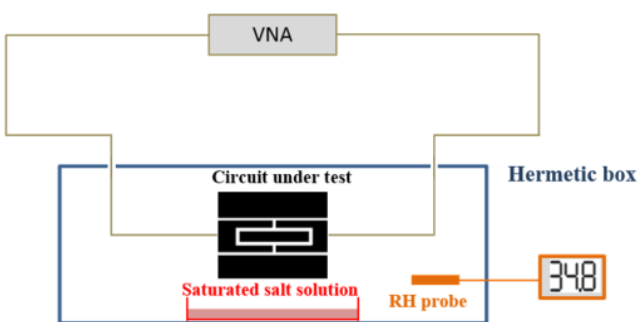

Fig. 12: Humidity test set-up schematic

principle. Moreover, the humidity response exhibits good linearity. The phase sensitivity is defined as the relative variation of the insertion phase with RH, as shown in Eq. (7):

$$
S_{\phi}=\frac{\Delta \phi_{21}}{\Delta(R H)}
$$

The measured overall phase variation was $\Delta \phi_{21}=8.40^{\circ}$ and RH variation of $\Delta(R H)=52.5 \% \mathrm{RH}$, leading to a sensitivity $S_{\phi}=0.154^{\circ} \%$ RH over the $22.8-75.3 \%$ RH range.

\section{DISCUSSION}

\section{A. Insertion Losses Analysis}

Fig. 13-b shows $S_{21}$ magnitude variation with RH. It appears insertion losses are improved as RH increases. Indeed, $\left|S_{21}\right|$ gradually moved from $5.63 \mathrm{~dB}$ at $22.8 \% \mathrm{RH}$ to $4.37 \mathrm{~dB}$ at $75.3 \% \mathrm{RH}$, that is a decrease of $1.26 \mathrm{~dB}$. The improvement in insertion losses agrees with the decrease of $R_{C}(R H)$ with $\mathrm{RH}$. The lower $R_{C}(R H)$ is, the better insertion losses are. To further minimize the final value of insertion losses, some avenues can be considered. One is the choice of sensing materials having a relatively high conductivity in ambient conditions. The fewer insertion losses are in ambient conditions, the fewer they will probably be after interaction with RH. Another clue is to properly match the sintering technique to sensitive material properties (e.g. thermal, photonic), and optimize the recipes.

\section{B. Phase Figure of Merit Analysis}

One definition of $F_{\Delta \phi}$ is the ratio of transmission phase variation to the mean value of insertion losses over the RH range $[5,40]$. Another definition considers the highest absolute value of insertion losses rather than the mean value [41]. However, these definitions are highly dependent on the insertion losses Lines with high insertion losses will always have poor figure of merit initial value, at the expense of phase shift.

Here, another definition of $F_{\Delta \phi}$ is introduced, with the thinking of evaluating to what extent the phase increases without reaching a critical attenuation of the EM signal. The proposed definition of $F_{\Delta \phi}$ is the variation of transmission phase $\left(\Delta \phi_{21}\right)$ ratio to the variation of insertion losses $\left(\Delta S_{21}\right)$, as given Eq. (8). Through this definition, $F_{\Delta \phi}$ measures the influence of the insertion losses worsening over the phase sensitivity. Higher $F_{\Delta \phi}$ is, better is the phase shifting paradigm operation.

$$
F_{\Delta \phi}=\left|\frac{\Delta \phi_{21}}{\Delta S_{21}}\right|=\left|\frac{\Delta \phi_{21} / \Delta(\mathrm{RH})}{\Delta S_{21} / \Delta(\mathrm{RH})}\right|=\left|\frac{S_{\phi}}{\Delta S_{21} / \Delta(\mathrm{RH})}\right|
$$




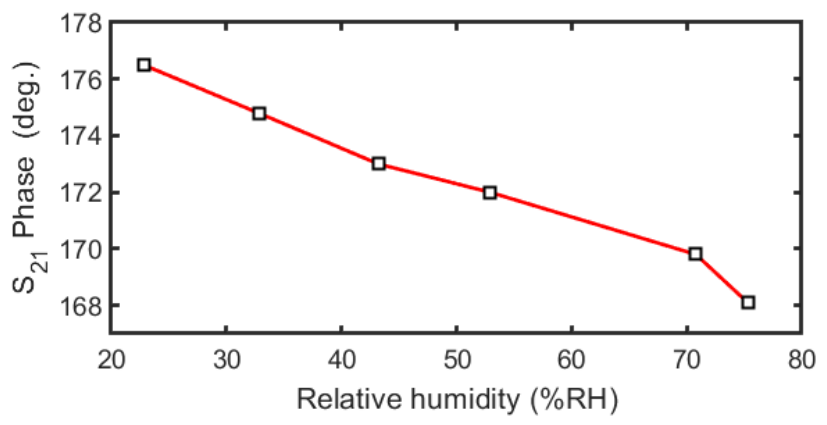

(a)

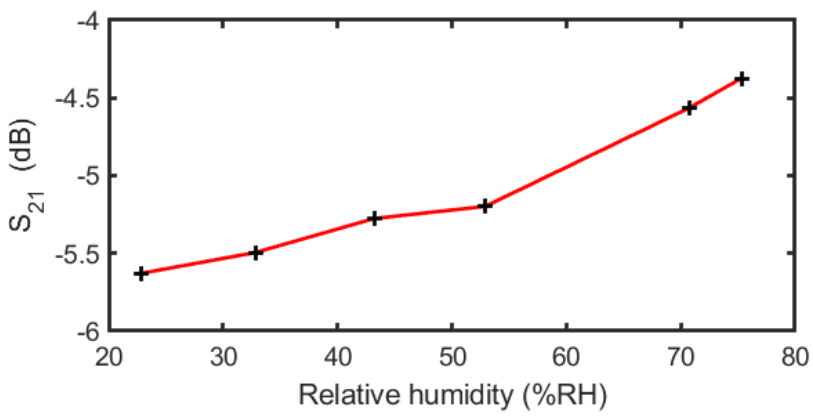

(b)

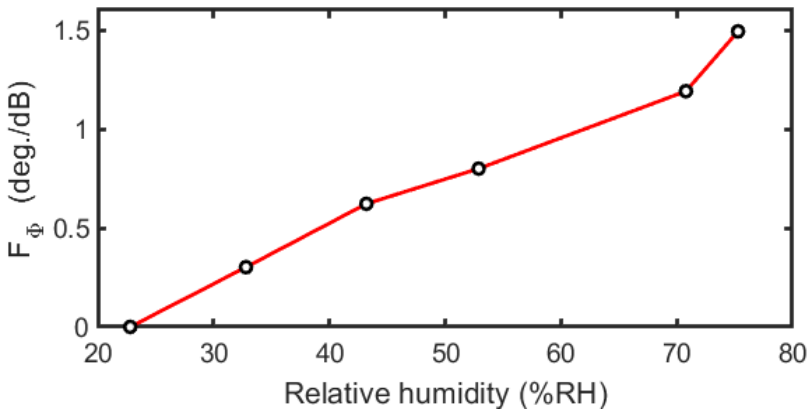

(c)

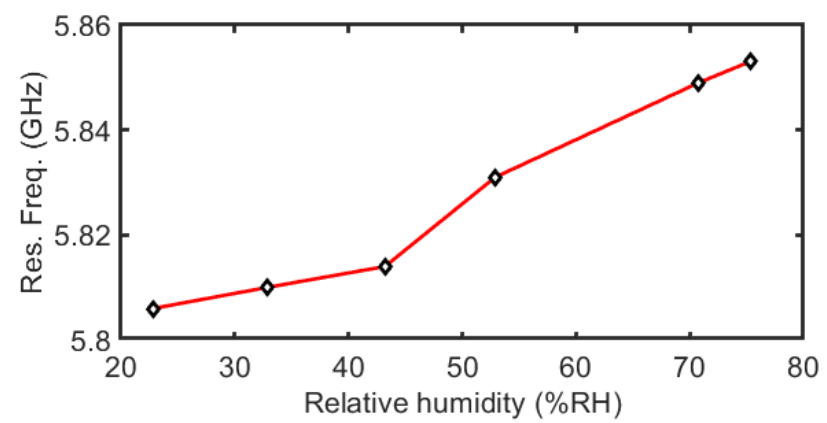

(d)

Fig. 13: Curves microwave parameters variation of the printed sensor as a function of RH (a) $S_{21}$ phase, (b) $S_{21}$ magnitude, (c) Phase figure of merit and (d) resonant frequency.

$F_{\Delta \phi}$ variation with RH was computed and plotted, as depicted in Fig. 13-c. $F_{\Delta \phi}$ increases as RH increases. The increase of $F_{\Delta \phi}$ is explained by the low insertion losses variation $\left(\Delta S_{21}=1.26\right.$ $\mathrm{dB}$ ) combined with the constant increase of phase transmission as RH increased $\left(\Delta \phi_{21}=8.40^{\circ}\right)$. From Fig. 13-c, the maximum value of the phase figure of merit was $F_{\Delta \phi}=6.7^{\circ} / \mathrm{dB}$. For comparison purposes, the maximum phase figure of merit as defined in [40] was measured at $F_{\Delta \phi}=1.50 \%$ dB. The gap can be explained by printing technologies characteristics, namely the low thickness of printing substrates. The proposed sensing principle is very efficient with printed devices.

\section{Resonance Shift and Return Loss Analysis}

From Fig. 13-d, a resonance shifting occurred as RH increases. The measured resonant frequency gradually changed from $5.806 \mathrm{GHz}$ at $22.8 \% \mathrm{RH}$ to $5.853 \mathrm{GHz}$ at $75.3 \% \mathrm{RH}$. This result is the experimental validation of the above-mentioned hypothesis in section IV-A. The overall frequency variation was $\Delta f_{r}=47 \mathrm{MHz}$, with only $3.5 \mathrm{MHz}$ variation in 22.5 - $52.9 \% \mathrm{RH}$. To explain difference above $52.9 \% \mathrm{RH}$, the hypothesis was made that TOCN/PPy layer might saturate from a threshold value of RH around $52.9 \%$ RH. Similar behaviour was observed with TOCN-based compounds in the literature [9]. Once saturated, the electrical conductivity of the inner rectangle increases rapidly, and the resonant frequency jumps.

It appears that return losses improve as RH increased according to Fig. 13-a. $\left|S_{11}\right|$ varied from $17.13 \mathrm{~dB}(22.8 \% \mathrm{RH})$ to $19.42 \mathrm{~dB}(75.3 \% \mathrm{RH})$. The difference is $2.29 \mathrm{~dB}$. This result agrees with theoretical expectations. As RH increases, $\sigma_{C}(R H)$ increases and $R_{C}(R H)$ decreases accordingly. The inner rectangle is less resistive, and the test structure input impedance $Z_{\text {in }}$ is optimized getting close to $50 \Omega$. Here, $Z_{\text {in }}$ value was computed to be $55.63 \Omega(22.8 \% \mathrm{RH})$ to $47.60 \Omega$ at $(70.8 \% \mathrm{RH})$ [42].

\section{COMPARISON WITH LITERATURE RESULTS}

The phase sensitivity in this paper was compared to previous results in the literature of microwave sensors operating with phase shifting sensing schemes.

Almost all the results were reported with rigid substrates such as FR4 or Rogers. Some have investigated other environmental parameters such as hydrogen [43] and temperature sensing [44]. For RH sensing, only the results on similar RH range were considered. The sensitivity varied from $0.004^{\circ} \% \mathrm{RH}$ with TOCN/PVOH sheet in 30-70 \%RH [45], to $0.18^{\circ} \% \mathrm{RH}$ with TEMPO oxidized thermomechanical pulp [7]. TOCN foils exhibited $0.025^{\circ} \% \mathrm{RH}$ on $0-70 \% \mathrm{RH}$ range [5], whereas TOCN and TOCN/PVOH inks reached $0.14^{\circ} / \% \mathrm{RH}$ and $0.11^{\circ} \% \mathrm{RH}$ respectively, in $22-70 \% \mathrm{RH}$ range [46]. The sensitivity with PEDOT-PSS ink was $0.14^{\circ} \%$ RH over $40-100 \%$ RH range [41]. The only result using printing technologies was $0.18^{\circ} \% \mathrm{RH}$ in $60-100 \% \mathrm{RH}$, with Kapton $\mathrm{HN}$ as a sensitive substrate.

The result of this paper is sometimes better than that of literature. Such performance is attributed to the efficiency of the sensing principle in the context of printing technologies. The thinness of printing susbtrates makes it possible for the conductive inks to impact the insertion phase and sensitivity notably.

\section{CONCLUSION}

This paper presented a passive CPW-based screen-printed humidity sensor operating through a phase shifting sensing scheme, and using TOCN/PPy electrically conductive film as sensing material. Simulation and experimental results validated this concept with a sensitivity of $0.154^{\circ} \% \mathrm{RH}$ in $22.8-75.3$ $\%$ RH range. Insertion losses increased by $1.26 \mathrm{~dB}$ and the figure of merit was $6.7^{\circ} / \mathrm{dB}$. The trade-off between the sensitivity and the insertion losses must be considered. 


\section{REFERENCES}

A. Alreshaid, J. Hester, W. Su, Y. Fang, and M. Tentzeris, "Ink-Jet Printed Wireless Liquid and Gas Sensors for IoT, SmartAg and Smart City Applications," Journal of The Electrochemical Society, vol. 165, no. 10, B407-B413, 2018

[2] K. W. Chun, H. Kim, and K. Lee, "A Study on Research Trends of Technologies for Industry 4.0; 3D Printing, Artificial Intelligence, Big Data, Cloud Computing, and Internet of Things," in Advanced Multimedia and Ubiquitous Engineering: Springer, 2018, 397-403.

[3] C. A. Pardue et al., "Design and Characterization of Inductors for Self-Powered IoT Edge Devices," IEEE Trans. Compon. Packag. Manuf. Technol., vol. 8, no. 7, 1263-1271, 2018

[4] T. K. Saha, T. N. Knaus, A. Khosla, and P. K. Sekhar, "Investigation of printing properties on paper substrate," Journal of The Electrochemical Society, vol. 165, no. 8, B3163-B3167, 2018.

[5] G. Ayissi Eyebe, B. Bideau, N. Boubekeur, É. Loranger, and F. Domingue, "Environmentally-friendly cellulose nanofibre sheets for humidity sensing in microwave frequencies," Sensors and Actuators B: Chemical, vol. 245, 484-492, 2017.

[6] Z. Chen and C. Lu, "Humidity sensors: a review of materials and mechanisms," Sensor letters, vol. 3, no. 4, 274-295, 2005. G. Ayissi Eyebe, D. Myja, B. Bideau, R. Lanouette, É. Loranger, and F. Domingue, "Coplanar waveguide techno TEMPO Oxidized Thermomechanical Pulp-Based Microwave Humidity Sensor," IEEE Sensors Letters, vol. 3, no. 7, 1-4, 2019.

M. Mikolajek, T. Reinheimer, N. Bohn, C. Kohler, M. J. Hoffmann, and J. R. Binder, "fabrication and characterization of fully inkjet printed capacitors Based on ceramic/polymer composite Dielectrics on flexible Substrates," Scientific reports, vol. 9, no. 1, 1-13, 2019. G. Ayissi Eyebe, N. Sama, N. Boubekeur, and F. Domingue, "Microwave Sensing Schemes of CPW Resonators Fully Printed on Humidity Sensitive Substrates," IEEE Microw. Wireless Compon. Lett., vol. 29, no. 4, 303-305, 2019.

D. M. Pozar, "Microwave engineering," Wiley, 2012.

Nikfalazar et al., "Inkjet printed BST thick-films for x-band phase shifter and phased array applications," IEEE International Workshop on Antenna Technology (iWAT), 121-124, 2013.

D. Kienemund et al., "Temperature dependence of a tunable phase shifter based on inkjet printing technology," IEEE German Microwave Conference, 150-153, 2015.

D. Lu, M. Maasch, A. Penirschke, Y. Zheng, C. Damm, and R. Jakoby, "Broadband Permittivity Characterization of PolyvinylAlcohol Film for Humidity Sensing Applications," IEEE Trans. Microw. Theory Techn., vol. 64, no. 10, 3255-3263, 2016.

A. Vena, L. Sydänheimo, M. M. Tentzeris, and L. Ukkonen, "A Fully Inkjet-Printed Wireless and Chipless Sensor for $\mathrm{CO} 2$ and Temperature Detection," IEEE Sensors Journal, vol. 15, no. 1, 8999, 2015 .

P. Bahoumina et al., "Microwave flexible gas sensor based on polymer multi wall carbon nanotubes sensitive layer," Sensors and Actuators B: Chemical, vol. 249, 708-714, 2017.

L. Xiang, Z. Wang, Z. Liu, S. E. Weigum, Q. Yu, and M. Y. Chen, "Inkjet-printed flexible biosensor based on graphene field effect transistor," IEEE Sensors Journal, vol. 16, no. 23, 8359-8364, 2016. T. Vuorinen, J. Niittynen, T. Kankkunen, T. M. Kraft, and M. Mäntysalo, "Inkjet-printed graphene/PEDOT: PSS temperature sensors on a skin-conformable polyurethane substrate," Scientific reports, vol. 6, 35289, 2016.

J. Fernández-Salmerón, A. Rivadeneyra, M. A. C. Rodríguez, L. F. Capitan-Vallvey, and A. J. Palma, "HF RFID Tag as Humidity Sensor: Two Different Approaches," IEEE Sensors Journal, vol. 15, no. 10, 5726-5733, 2015.

M. Yasir, S. Bistarelli, A. Cataldo, M. Bozzi, L. Perregrini, and S. Bellucci, "Tunable Phase Shifter Based on Few-Layer Graphene Flakes," IEEE Microw. Wireless Compon. Lett., vol. 29, no. 1, 4749, 2019.

H. Deng and F. Lin, "Continuously Tunable True- Time- Delay Phase Shifter Using Switchable Varactor- Tuned Transmission Lines," IEEE/MTT-S International Microwave Symposium, 10-15, 514-517, 2018

F. Lin and H. Deng, "Continuously Tunable True-Time-Delay Phase Shifter Based on Transmission Lines With Simultaneously Reconfigurable Impedance and Phase Constant," IEEE Trans. Microw. Theory Techn., vol. 67, no. 12, 4714-4723, 2019.
K. C. Gupta, R. Garg, and I. J. Bahl, Microstrip Lines and Slotlines. Artech House, 1979.

www.novacentrix.com

M. Konieczna, E. Markiewicz, and J. Jurga, "Dielectric properties of polyethylene terephthalate/polyphenylene sulfide/barium titanate nanocomposite for application in electronic industry," Polymer Engineering \& Science, vol. 50, no. 8, 1613-1619, 2010.

K. Hettak, N. Dib, A. F. Sheta, and S. Toutain, "A class of novel uniplanar series resonators and their implementation in original applications," IEEE Microw. Theory Techn., vol. 46, no. 9, 12701276,1998

R. N. Simons, Coplanar waveguide circuits, components, and systems. John Wiley \& Sons, 2004.

B. C. Wadell, Transmission Line Design Handbook. Artech House, 1991. using the space domain integral equation method (SDIE)," Journal of Electromagnetic waves and Applications, vol. 5, no. 4-5, 503$523,1991$.

[29] N. I. Dib, Theoretical characterization of coplanar waveguide transmission lines and discontinuities. University of Michigan, 1992.

[30] G. Ayissi Eyebe, B. Bideau, É. Loranger, and F. Domingue, "Fully printed CPW-based microwave humidity sensor in phase shifting paradigm," IEEE International Conference on Flexible and Printable Sensors and Systems (FLEPS), 1-3, 2019.

[31] X. Leng, W. Li, D. Luo, and F. Wang, "Differential structure with graphene oxide for both humidity and temperature sensing," IEEE Sensors Journal, vol. 17, no. 14, 4357-4364, 2017.

[32] A. Bijalwan and V. Rastogi, "Design and Simulation of a PalladiumAluminum Nanostructure based Hydrogen Sensor with Improved Figure of Merit," IEEE Sensors Journal, 2019.

[33] T. Zhang et al., "Analysis of de and ac properties of humidity sensor based on polypyrrole materials," Sensors and Actuators B: Chemical, vol. 131, no. 2, 687-691, 2008.

[34] S. K. Mahadeva, S. Yun, and J. Kim, "Flexible humidity and temperature sensor based on cellulose-polypyrrole nanocomposite," Sensors and Actuators A: Physical, vol. 165, no. 2, 194-199, 2011.

[35] W.-D. Lin, H.-M. Chang, and R.-J. Wu, "Applied novel sensing material graphene/polypyrrole for humidity sensor," Sensors and Actuators B: Chemical, vol. 181, 326-331, 2013.

[36] S. K. Shukla, "Synthesis and characterization of polypyrrole grafted cellulose for humidity sensing," International Journal of Biological Macromolecules, vol. 62, 531-536, 2013.

[37] H. C. Kang and K. E. Geckeler, "Enhanced electrical conductivity of polypyrrole prepared by chemical oxidative polymerization: effect of the preparation technique and polymer additive," Polymer, vol. 41, no. 18, 6931-6934, 2000.

[38] T.-M. Wu, H.-L. Chang, and Y.-W. Lin, "Synthesis and characterization of conductive polypyrrole with improved conductivity and processability," Polymer International, vol. 58, no. 9, 1065-1070, 2009.

[39] L. Greenspan, "Humidity fixed points of binary saturated aqueous solutions," Journal of research of the national bureau of standards, vol. 81, no. 1, 89-96, 1977.

[40] O. Vendik, I. Vendik, and M. Nikol'ski, "Comparison of MMIC phase shifters using figure of merit as the main characteristic," International Conference on Microwaves, Radar and Wireless Communications (IEEE Cat. No. 04EX824), vol. 1, 333-335, 2004.

[41] M. C. Caccami, S. Manzari, and G. Marrocco, "Phase-Oriented Sensing by Means of Loaded UHF RFID Tags," IEEE Trans. Antennas Propag., vol. 63, no. 10, 4512-4520, 2015.

[42] G. Ayissi Eyebe, "Conception et réalisation de capteurs micro-ondes sans fil par les technologies d'impression," Université du Québec à Trois-Rivières, 2018.

[43] A. Benleulmi, N. Y. Sama, P. Ferrari, and F. Domingue, "Substrate integrated waveguide phase shifter for hydrogen sensing," IEEE Microwav. Wireless Compon. Lett., vol. 26, no. 9, 744-746, 2016.

[44] G. Ayissi Eyebe, A. H. Rasolomboahanginjatovo, B. Bideau, and F. Domingue, "Investigation on temperature-dependent dielectric properties of ETFE fluoropolymer for microwave temperature sensing applications," Sensors and Actuators A: Physical, vol. 290, 215-221, 2019.

[45] G. Ayissi Eyebe, B. Bideau, E. Loranger, N. Boubekeur, and F. Domingue, "Novel TOCN/PVOH dielectric composite sheets with 
low ecological footprint for microwave humidity sensing," IEEE sensors letters, vol. 2, no. 4, 1-4, 2018.

[46] G. A. Eyebe, B. Bideau, É. Loranger, and F. Domingue, "TEMPOoxidized cellulose nanofibre (TOCN) films and composites with $\mathrm{PVOH}$ as sensitive dielectrics for microwave humidity sensing," Sensors and Actuators B: Chemical, vol. 291, 385-393, 2019. 